\title{
Color Lie algebras and Lie algebras of order $F$
}

\author{
R. Campoamor-Stursberg ${ }^{* a}$, M. Rausch de Traubenberg ${ }^{\dagger b}$ \\ ${ }^{a}$ Instituto de Matemática Interdisciplinar, \\ Universidad Complutense de Madrid, 3 Plaza de Ciencias, 28040 Madrid, Spain. \\ ${ }^{b}$ Laboratoire de Physique Théorique, CNRS UMR 7085, Université Louis Pasteur \\ 3 rue de l'Université, 67084 Strasbourg Cedex, France
}

November 20, 2008

\begin{abstract}
The notion of color algebras is generalized to the class of $F$-ary algebras, and corresponding decoloration theorems are established. This is used to give a construction of colored structures by means of tensor products with Clifford-like algebras. It is moreover shown that color algebras admit realisations as $q=0$ quon algebras.
\end{abstract}

2000 MSC: 17B70, 17B75, 17B81

\section{Introduction}

The problem of finding mass formulae for particles belonging to a representation of an interaction group motivated, in the beginning sixties, the efforts to combine interactions with relativistic invariance in a non-trivial way. The well known obstructions to such a construction [3] finally led to the supersymmetric schemes. In this sense, two different models unifying internal and space-time symmetries, the conformal superalgebras $\mathfrak{s u}(2,2 ; N)$ and the orthosymplectic algebra $\mathfrak{o} \mathfrak{s p}(4 ; N)$ were proposed [6]. The introduction of a grading was soon recognized to be an indispensable requirement to introduce transformations relating states obeying different quantum statistics types. However, a further generalization seemed necessary to clearly distinguish the color and flavor degrees of freedom, which were treated in the same way in the two previous models. A first approach to this question was made in [11], where a color algebra based on the nonassociative octonions was proposed. This scheme constituted a mathematical model taking into account the unobservability of quarks and their associated massless color gauge bosons. An alternative construction, preserving the associative framework, was given in [17]. These structures motivated by themselves the study of several extensions of Lie algebras, keeping in mind the main properties that made them interesting to describe symmetries of physical phenomena. Among others, the generalizations that have been proven to be physically relevant are color (or graded) Lie algebras $[2,10,13,17,19]$ and, more recently, Lie algebras of order $F[9,14]$. These two types of algebras share some properties, and are based upon a grading by an Abelian group.

Tensor products constitute a natural tool to construct higher dimensional algebraic objects starting from two given ones, as well as to study their representation theory and the underlying Clebsch-Gordan problem. However, the tensor product of two algebras usually give rise to non-trivial identities that must be satisfied, and often fail to preserve certain key properties (as happens e.g. for Lie algebras). In order to prevent this situation, generalized tensor products have been developed for various structures, such as groups or Lie (super)algebras. In a more general frame, there is no reason to believe that tensor products of algebras

\footnotetext{
*e-mail:rutwig@pdi.ucm.es

$\dagger$ e-mail: rausch@lpt1.u-strasbg.fr
} 
with different structures can not lead to further interesting structures, possibly preserving some of the main properties of its components. Some attention has been devoted, in this direction, to tensor products of the type $C \otimes D$, where $C$ is a Clifford algebra $C$ and $D$ a $\mathbb{Z}_{2}$-graded ring of differential operators on a manifold, where the differential operators are interpreted, in some sense, as "quantum mechanical", the classical approximation of which is given by a Poisson bracket. These products suggest a deep relation with the commutation-anticommutation formalism in Field Theory [4].

In this paper we show that color algebras and Lie algebras of order $F$ can be unified leading to some new algebras that we call color Lie (super)algebra of order $F$. Furthermore, we show that many examples of these algebras can be seen as tensor product of given algebras. In Section 2 we recall some results concerning the general theory of color algebras, focusing on the isomorphism between color and graded algebras [1]. We also review the main features of a distinguished class of $F$-ary algebra. Section 4 is devoted to give a unification of both mentioned types of structures, as well as an adapted decoloration theorem. It turns out that color algebras arise as tensor products of ordinary non-color algebras with algebras of Clifford type. In the last section we show that all considered types of algebras are strongly related to quon algebras for $q=0$. In particular, "differential" realisations in terms of quon algebras are obtained.

\section{Color Lie algebras}

Color Lie (super)algebras, originally introduced in [17], can be seen as a direct generalization of Lie (super)algebras. Indeed, the latter are defined through antisymmetric (commutator) or symmetric (anticommutator) products, although for the former the product is neither symmetric nor antisymmetric and is defined by means of a commutation factor. This commutation factor is equal to \pm 1 for (super)Lie algebras and more general for arbitrary color Lie (super)algebras.

As happened for Lie superalgebras, the basic tool to define color Lie (super)algebras is a grading determined by an Abelian group. The latter, besides defining the underlying grading in the structure, moreover provides a new object known as commutation factor.

Definition 2.1 Let $\Gamma$ be an abelian group. A commutation factor $N$ is a map $N: \Gamma \times \Gamma \rightarrow \mathbb{C} \backslash\{0\}$ satisfying the following constraints:

1. $N(a, b) N(b, a)=1$, for all $a, b \in \Gamma$;

2. $N(a, b+c)=N(a, b) N(a, c)$, for all $a, b, c \in \Gamma$;

3. $N(a+b, c)=N(a, c) N(b, c)$, for all $a, b, c \in \Gamma$.

The definition above implies, in particular, the following relations

$$
N(0, a)=N(a, 0)=1, \quad N(a, b)=N(-b, a), \quad N(a, a)= \pm 1 \text { for all } a, b \in \Gamma,
$$

where 0 denotes the identity element of $\Gamma$. In particular, fixed one element of $\Gamma$, the induced mapping $N_{x}: \Gamma \rightarrow \mathbb{C} \backslash\{0\}$ defines a homomorphism of groups.

Definition 2.2 Let $\Gamma$ be an abelian group and $N$ be a commutation factor. The (complex) graded vector space $\mathfrak{g}=\underset{a \in \Gamma}{\bigoplus} \mathfrak{g}_{a}$ is called a color Lie (super)algebra if

1. $\mathfrak{g}_{0}$ is a (complex) Lie algebra.

2. For all $a \in \Gamma \backslash\{0\}, \mathfrak{g}_{a}$ is a representation of $\mathfrak{g}_{0}$. If $X \in \mathfrak{g}_{0}, Y \in \mathfrak{g}_{1}$ then $[|X, Y|]_{N}=[X, Y]$ denotes the action of $X$ on $Y$.

3. For all $a, b \in \Gamma$, there exists a $\mathfrak{g}_{0}-$ equivariant map $[|,|]_{N} \mathfrak{g}_{a} \times \mathfrak{g}_{b} \rightarrow \mathfrak{g}_{a+b}$ such that for all $X \in \mathfrak{g}_{a}, Y \in \mathfrak{g}_{b}$ the constraint $[|X, Y|]_{N}=-N(a, b)[|Y, X|]_{N}$ is satisfied. 
4. For all $X \in \mathfrak{g}_{a}, Y \in \mathfrak{g}_{b}, Z \in \mathfrak{g}_{c}$, the following Jacobi identities

$$
\left[\left|X,[|Y, Z|]_{N}\right|\right]_{N}=\left[\left|[|X, Y|]_{N}, Z\right|\right]_{N}+N(a, b)\left[\left|Y,[|X, Z|]_{N}\right|\right]_{N}
$$

hold.

Remark 2.3 The Jacobi identity above can be rewritten in equivalent form as

$$
N(c, a)\left[\left|X,[|Y, Z|]_{N}\right|\right]_{N}+N(a, b)\left[\left|Y,[|Z, X|]_{N}\right|\right]_{N}+N(c, a)\left[\left|Z,[|Y, X|]_{N}\right|\right]_{N}=0 .
$$

Further, property 1 in definition 2.1 is a consequence of 3 in definition 2.2, while property 2 in Definition 2.1 is a consequence of the Jacobi identity 4 in Definition 2.2. For the particular case $\Gamma=\{0\}, \mathfrak{g}=\mathfrak{g}_{0}$ reduces to a Lie algebra. If $\Gamma=\mathbb{Z}_{2}$, we obtain the grading $\mathfrak{g}=\mathfrak{g}_{0} \oplus \mathfrak{g}_{1}$. If in addition $N(1,1)=-1$ holds, $\mathfrak{g}$ is just a Lie superalgebra. Therefore, the latter condition in definition 2.2 points out to which extent color algebras extend ordinary Lie algebras and superalgebras. Furthermore, if $N(a, b)=1$ for all $a, b \in \Gamma, \mathfrak{g}$ is $a$ Lie algebra graded by the group $\Gamma$. From now Lie algebras with this last property will be called $\Gamma$-graded Lie algebras.

The grading group $\Gamma$ inherits naturally a $\mathbb{Z}_{2}$-grading: $\Gamma=\Gamma_{0} \oplus \Gamma_{1}$, where $\Gamma_{i}=\left\{a \in \Gamma \mid N(a, a)=(-1)^{i}\right\}$ [19]. If $\Gamma_{1}=0\left(\Gamma_{1} \neq 0\right), \mathfrak{g}$ is called a color Lie algebra (respectively superalgebra). Starting from a color Lie superalgebra, we define $N_{+}$by $N_{+}(a, b)=(-1)^{|a||b|} N(a, b)$, where $|a|$ is the degree of $a$ with respect to the $\mathbb{Z}_{2}$-grading. It is not difficult to check that $N_{+}$is also a commutation factor. Furthermore, if we decompose $\mathfrak{g}=\mathfrak{g}_{0} \oplus \mathfrak{g}_{1}$ with respect to this $\mathbb{Z}_{2}$-grading, and introduce the Grassmann algebra $\Lambda\left(\mathbb{C}^{m}\right)$, the analogue of the Grassmann-hull in the case of Lie superalgebras, we can endow the color Lie superalgebra with a color Lie algebra structure. Indeed, if we set $\Lambda\left(\mathbb{C}^{m}\right)=\Lambda\left(\mathbb{C}^{m}\right)_{0} \oplus \Lambda\left(\mathbb{C}^{m}\right)_{1}$, where $\Lambda\left(\mathbb{C}^{m}\right)_{i}$ are of degree $i$ then

$$
\left(\Lambda\left(\mathbb{C}^{m}\right) \otimes \mathfrak{g}\right)_{0}=\Lambda\left(\mathbb{C}^{m}\right)_{0} \otimes \mathfrak{g}_{0} \oplus \Lambda\left(\mathbb{C}^{m}\right)_{1} \otimes \mathfrak{g}_{1},
$$

is a color Lie algebra with commutation factor $N_{+}$.

Remark 2.4 To any associative $\Gamma$-graded algebras $\mathcal{A}=\underset{a \in \Gamma}{\oplus} \mathcal{A}_{a}$ with multiplication law $\mu$, one can associate a color Lie (super)algebra with commutation factor $N$ denoted $\mathcal{A}_{N}$ by means of

$$
\left[\left|X_{1}, X_{2}\right|\right]_{N}=\mu\left(X_{1}, X_{2}\right)-N(a, b) \mu\left(X_{2}, X_{1}\right), \text { for any }(X, Y) \in \mathcal{A}_{a} \times \mathcal{A}_{b}
$$

On can easily see that the Jacobi identities are a consequence of the associativity of the product $\mu$.

Introducing a graded basis $\left\{T_{i}^{(a)}, i=1, \cdots, \operatorname{dim} \mathfrak{g}_{a}\right\}$ of $\mathfrak{g}_{a}, a \in \Gamma$, the commutator is expressed as

$$
\left[\left|T_{\alpha}^{(a)}, T_{\beta}^{(b)}\right|\right]_{N}=C^{(a)(b)}{ }_{\alpha \beta}^{\gamma} T_{\gamma}^{(a+b)} .
$$

The scalars $C^{(a)(b)}{ }_{\alpha \beta}^{\gamma}$ are called the structure constants of $\mathfrak{g}$ over the given basis.

Definition 2.5 A representation of a color Lie (super)algebra is a mapping $\rho: \mathfrak{g} \rightarrow \operatorname{End}(V)$, where $V=$ $\underset{a \in \Gamma}{\oplus} V_{a}$ is a graded vector space such that

$$
[|\rho(X), \rho(Y)|]_{N}=\rho(X) \rho(Y)-N(a, b) \rho(Y) \rho(X),
$$

for all $X \in \mathfrak{g}_{a}, Y \in \mathfrak{g}_{b}$.

We observe that for all $a, b \in \Gamma$ we have $\rho\left(\mathfrak{g}_{a}\right) V_{b} \subseteq V_{a+b}$, which implies that any $V_{a}$ has the structure of a $\mathfrak{g}_{0}$-module. Fixing an element $v \in V$ and denoting by $v_{i}(i \in I)$ its components, we can introduce the mapping gr $: I \rightarrow \Gamma$ defined by $i \mapsto \operatorname{gr}(i)$, from which we conclude that $V_{a}=\left\{v=\left(v_{i}, i \in I\right), \operatorname{gr}(i)=a\right\}$. The mapping gr is called the grading map. Now, for a given matrix representation $\rho\left(T^{(a)}{ }_{\alpha}\right)=M^{(a)}{ }_{\alpha}$, where $T^{(a)}{ }_{\alpha} \in \mathfrak{g}_{a}$, the non-vanishing indices of the matrix $M^{(a)}{ }_{\alpha}$ are those $\left(M^{(a)}{ }_{\alpha}\right)_{i}{ }^{j}$ satisfying the equality $\operatorname{gr}(i)-\operatorname{gr}(j)=a$. 
Example 2.6 Let $m=m_{1}+\cdots+m_{n}, V=\mathbb{C}^{m}$ and $\Gamma=\left\{a_{1}, \cdots, a_{n}\right\}$ be an abelian group of order $n$. Let gr be defined by

$$
\begin{array}{lll}
\operatorname{gr}(i)=a_{1} & & i=1, \cdots, m_{1} \\
\operatorname{gr}(i)=a_{2} & & i=m_{1}+1, \cdots, m_{1}+m_{2} \\
& \vdots & \\
\operatorname{gr}(i)=a_{k} & & i=m_{1}+\cdots+m_{k-1}+1, \cdots, m_{1}+\cdots+m_{k} \\
& \vdots & \\
\operatorname{gr}(i)=a_{n} & & i=m_{1}+\cdots+m_{n-1}+1, \cdots, m .
\end{array}
$$

Consider a commutation factor $N$ satisfying the previous relations (2.1). We construct the color algebra (H. Green and P. Jarvis in [10]) $\mathfrak{g r}\left(\{m\}_{\Gamma, N}\right)=\underset{a \in \Gamma}{\mathfrak{g}_{a}}$ by means of its defining relations. A basis of $\mathfrak{g}_{a}$ is given by the $m \times m$ complex matrices $\left(E^{p}{ }_{q}\right)_{i}{ }^{j}=\delta^{p}{ }_{i} \delta_{q}{ }^{j}, 1 \leq p, q \leq m$ with $\operatorname{gr}(q)-\operatorname{gr}(p)=a$. The space $\mathfrak{g}$ is endowed with a color Lie (super)algebra structure:

$$
\begin{aligned}
{\left[\left|E^{p}{ }_{q}, E^{r}{ }_{s}\right|\right]_{N} } & =E^{p}{ }_{q} E^{r}{ }_{s}-N(\operatorname{gr}(q)-\operatorname{gr}(p), \operatorname{gr}(s)-\operatorname{gr}(r)) E^{r}{ }_{s} E^{p}{ }_{q} \\
& =\delta^{r}{ }_{q} E^{p}{ }_{s}-N(\operatorname{gr}(q)-\operatorname{gr}(p), \operatorname{gr}(s)-\operatorname{gr}(r)) \delta^{p}{ }_{s} E^{r}{ }_{q} .
\end{aligned}
$$

Several subalgebras of $\mathfrak{g l}\left(\{m\}_{\Gamma, N}\right)$ can also be defined using this procedure (see [19, 10]). In particular, if $\mathfrak{g}$ is a color Lie (super)algebra with basis $\left\{X_{\alpha}, \alpha=1, \cdots, \operatorname{dim} \mathfrak{g}\right\}$ satisfying $\left[\left|X_{\alpha}, X_{\beta}\right|\right]_{N}=C_{\alpha \beta}{ }^{\gamma} X_{\gamma}, \mathfrak{g}$ can be embedded into some $\mathfrak{g l}(\{m\})_{\Gamma, N}$ if we define $X_{a}=C_{a b}{ }^{c} \bar{e}^{b}{ }_{c}$, where the $\bar{e}^{b}{ }_{c}$ satisfy $\left[\left|\bar{e}^{a}{ }_{b}, \bar{e}^{c}{ }_{d}\right|\right]_{N}=$ $\delta^{a}{ }_{d} \bar{e}^{c}{ }_{b}-N(\operatorname{gr}(b)-\operatorname{gr}(a), \operatorname{gr}(d)-\operatorname{gr}(c)) \delta^{c}{ }_{b} \bar{e}^{a}{ }_{d}$.

Example 2.7 Let $\mathfrak{g}_{0}$ be an arbitrary Lie algebra and let $\left\{T_{\alpha}, \alpha=1, \cdots, \operatorname{dim} \mathfrak{g}_{0}\right\}$ be a basis of $\mathfrak{g}_{0}\left(\left[T_{\alpha}, T_{\beta}\right]=\right.$ $\left.f_{\alpha \beta}{ }^{\gamma} T_{\gamma}\right)$. Consider $\mathcal{C}_{n}^{2}$, the complex algebra generated by $e_{1}, e_{2}$ such that

$$
e_{1}^{n}=e_{2}^{n}=1, e_{1} e_{2}=q e_{2} e_{1}, q=\exp \left(\frac{2 i \pi}{n}\right) .
$$

This structure, called the generalised Clifford algebra, has been studied by several authors (see [15] and references therein). Introduce $e_{a, b}=e_{1}^{a} e_{2}^{b}$ a basis of $\mathcal{C}_{n}^{2}$. It is easy to see that we have $e_{a, b} e_{c, d}=q^{-b c} e_{a+c, b+d}$, and thus $\mathfrak{g}=\mathcal{C}_{n}^{2} \otimes \mathfrak{g}_{0}$ is a color Lie algebra for which the abelian group is $\Gamma=\mathbb{Z}_{n} \times \mathbb{Z}_{n}$ and the commutation factor is $N((a, b),(c, d))=q^{a d-b c}$. Indeed, if we set $\mathfrak{g}_{a, b}=\left\{T_{\alpha}^{(a, b)}=e_{a, b} \otimes T_{\alpha}\right\}$ we have

$$
\left[\left|T_{\alpha}^{(a, b)}, T_{\beta}^{(c, d)}\right|\right]_{N}=q^{-b c} f_{\alpha \beta}{ }^{\gamma} T_{\gamma}^{(a+c, b+d)} .
$$

It is a matter of a simple calculation to check the Jacobi identities. Furthermore, it is known that $\mathcal{C}_{n}^{2}$ admits a unique irreducible $n \times n$ faithful matrix representation:

$$
\rho\left(e_{1}\right)=\sigma_{1}=\left(\begin{array}{ccccc}
0 & 1 & & \ldots & 0 \\
0 & 0 & 1 & \ldots & 0 \\
\vdots & & & \ddots & \vdots \\
0 & \ldots & 0 & \ldots & 1 \\
1 & \ldots & 0 & \ldots & 0
\end{array}\right), \rho\left(e_{2}\right)=\sigma_{2}=\left(\begin{array}{cccc}
1 & 0 & \ldots & 0 \\
0 & q & \ldots & 0 \\
\vdots & & \ddots & \vdots \\
0 & \ldots & 0 & q^{n-1}
\end{array}\right)
$$

If we introduce now the $n^{2} \times n^{2}$ matrices

$$
\rho_{1}=\sigma_{1} \otimes \mathbf{1}, \rho_{2}=\sigma_{2} \otimes \sigma_{1}
$$

and define $\rho_{(a, b)}=\rho_{1}^{a} \rho_{2}^{b}$, together with a $d$-dimensional matrix representation of $\mathfrak{g}_{0}$ given by $\rho\left(T_{\alpha}\right)=M_{\alpha}$, we obtain a $n^{2} d$ dimensional representation of $\mathfrak{g}: \rho\left(T_{\alpha}^{(a, b)}\right)=\rho_{(a, b)} \otimes M_{\alpha}$.

This construction can be extended for larger abelian groups. Indeed, starting from the generalised Clifford algebra $\mathcal{C}_{n}^{p}$ generated by $e_{1}, \cdots, e_{p}$ satisfying $e_{i}^{n}=1, i=1, \cdots, p, e_{i} e_{j}=q e_{j} e_{1}, 1 \leq i<j \leq p$ and defining $\rho_{a_{1}, \cdots, a_{p}}=e_{1}^{a_{1}} \cdots e_{p}^{a_{p}}$, we obtain in the same way a color Lie algebra with abelian group $\mathbb{Z}_{n}^{p}$. Finally, let 
us mention that a similar construction can also be obtained in straightforward way by starting from a Lie superalgebra.

A somewhat different ansatz, which turns out to be of wide interest in applications, refers to the realization of color Lie (super)algebra in terms of differential operators [10]. Let $\mathfrak{g}$ be a color Lie (super)algebra with basis $\left\{T_{\alpha}, \alpha=1, \cdots, \operatorname{dim} \mathfrak{g}\right\}$ and commutation relations $\left[\left|T_{\alpha}, T_{\beta}\right|\right]_{N}=C_{\alpha \beta}{ }^{\gamma} T_{\gamma}$. Denote by $N$ the commutation factor. Assume further that we have a $d$-dimensional matrix representation $\rho\left(T_{a}\right)=M_{a}$, and introduce $d$ variables $\theta^{i}$ together with their associated differential operators $\partial_{i}$. As before, we assume that the index $i$ is of degree $\operatorname{gr}(i), \theta^{i}$ is of degree $-\operatorname{gr}(i)$ and $\partial_{i}$ of degree $\operatorname{gr}(i)$ subjected to the following commutations relations

$$
\begin{aligned}
{\left[\left|\theta^{i}, \theta^{j}\right|\right]_{N} } & =\theta^{i} \theta^{j}+\epsilon N(\operatorname{gr}(i), \operatorname{gr}(j)) \theta^{j} \theta^{i}=0 \\
{\left[\left|\partial_{i}, \partial_{j}\right|\right]_{N} } & =\partial_{i} \partial_{j}+\epsilon N(\operatorname{gr}(i), \operatorname{gr}(j)) \partial_{j} \partial_{i}=0 \\
{\left[\left|\partial_{i}, \theta^{j}\right|\right]_{N} } & =\partial_{i} \theta^{j}+\epsilon N(\operatorname{gr}(i),-\operatorname{gr}(j)) \theta^{j} \partial_{i}=\delta_{i}{ }^{j}
\end{aligned}
$$

with $\epsilon= \pm 1$. A very elegant construction of $\theta^{i}$ and $\partial_{i}$ can be found in [12] in terms of usual bosons (when $\epsilon=-1$ ) or fermions (when $\epsilon=1$ ). If we set $\mathcal{M}_{a}=\theta^{i}\left(M_{a}\right)_{i}{ }^{j} \partial_{j}$ and suppose that $\operatorname{gr}(a)=\operatorname{gr}(j)-\operatorname{gr}(i), \operatorname{a}$ direct computation gives

$$
\begin{aligned}
{\left[\left|\mathcal{M}_{\alpha}, \theta^{i}\right|\right]_{N} } & =\theta^{j}\left(M_{\alpha}\right)_{j}{ }^{i} \\
{\left[\left|\mathcal{M}_{\alpha}, \partial_{i}\right|\right]_{N} } & =-N(\operatorname{gr}(j)-\operatorname{gr}(i), \operatorname{gr}(i))\left(M_{\alpha}\right)_{i}{ }^{j} \partial_{j}, \\
{\left[\left|\mathcal{M}_{\alpha}, \mathcal{M}_{\beta}\right|\right]_{N} } & =C_{\alpha \beta}{ }^{\gamma} \mathcal{M}_{\gamma} .
\end{aligned}
$$

This means that the variables $\theta^{i}$ are in the fundamental representation of $\mathfrak{g}_{a}$, while the variables $\partial_{i}$ belong to the corresponding dual representation. These two sets of variables, generalizing the usual bosonic and fermionic algebras, play a central role in differential realizations of $\mathfrak{g}_{a}$. The next result shows that to a color Lie algebra, we can associate a graded Lie algebra with the same grading group $\Gamma$. For this reason we call it decoloration theorem.

Proposition 2.8 There is an isomorphism between color Lie (super)algebras and graded Lie (super)algebras.

Proof. Consider a color Lie (super)algebra $\mathfrak{g}=\bigoplus_{a \in \Gamma} \mathfrak{g}_{a}=\bigoplus_{a \in \Gamma}\left\langle T_{\alpha}^{(a)}, \alpha=1, \cdots, \operatorname{dim} \mathfrak{g}_{a}\right\rangle$ with commutation factor $N$ and grading group $\Gamma$. We also introduce the commutation factor $N_{+}$as defined previously. In the case where $\mathfrak{g}$ is a color Lie algebra we have $N_{+}=N$. Consider now a graded algebra $G=\underset{a \in \Gamma}{\oplus} G_{a}$ with $G_{a}=\mathbb{C} e_{a}$ and multiplication law given by

$$
e_{a} e_{b}=\sigma(a, b) e_{a+b}
$$

such that the following constraint is satisfied [19]:

$$
\sigma(a, b+c) \sigma(b, c)=\sigma(a, b) \sigma(a+b, c), \forall a, b, c \in \Gamma
$$

If we suppose that $\sigma(a, b) \sigma^{-1}(b, a)=N_{+}^{-1}(a, b)$ holds, then we have the equality $e_{-a} e_{-b}-N_{+}^{-1}(a, b) e_{-b} e_{-a}=$ 0 . This implies that $G$ is a subalgebra of the associative algebra defined by equations (2.3). As a consequence, condition (2.6) is equivalent to assume the associativity of the product in $G$. If we further suppose that $N_{+}$ is a commutation factor, the additional condition

$$
\sigma(a, b+c) \sigma^{-1}(a, b) \sigma^{-1}(a, c)=\sigma(b+c, a) \sigma^{-1}(b, a) \sigma^{-1}(c, a), \forall a, b, c \in \Gamma
$$


is satisfied. We call $\sigma$ a multiplier. In fact, it can be easily shown that equation (2.7) is a consequence of (2.6). Let us define

$$
\tilde{\mathfrak{g}}=\underset{a \in \Gamma}{\oplus} \tilde{\mathfrak{g}}_{a}=\underset{a \in \Gamma}{\bigoplus_{-}} e_{-a} \otimes \mathfrak{g}_{a} .
$$

We observe that all elements in $\tilde{\mathfrak{g}}_{a}$ (for any $a \in \Gamma$ ) are of degree zero. For $X \in \mathfrak{g}_{a}, Y \in \mathfrak{g}_{b}$ we set $\tilde{X}=$ $e_{-a} \otimes X, \tilde{Y}=e_{-b} \otimes Y \in \tilde{\mathfrak{g}}_{a}, \tilde{\mathfrak{g}}_{b}$. From this, we derive the commutators

$$
[\tilde{X}, \tilde{Y}]_{ \pm}=\sigma(-a,-b) e_{-a-b} \otimes[|X, Y|]_{N} \in \tilde{\mathfrak{g}}_{a+b}
$$

These new brackets (2.8) satisfy the Jacobi identity (for $\tilde{X}=e_{-a} \otimes X, \tilde{Y}=e_{-b} \otimes Y, \tilde{Z}=e_{-c} \otimes Z \in \tilde{\mathfrak{g}}_{a}, \tilde{\mathfrak{g}}_{b}, \tilde{\mathfrak{g}}_{c}$ )

$$
(-1)^{|\tilde{Z}||\tilde{X}|}\left[\tilde{X},[\tilde{Y}, \tilde{Z}]_{ \pm}\right]_{ \pm}+(-1)^{|\tilde{X}||\tilde{Y}|}\left[\tilde{Y},[\tilde{Z}, \tilde{X}]_{ \pm}\right]_{ \pm}+(-1)^{|\tilde{Y}||\tilde{Z}|}\left[\tilde{Z},[\tilde{X}, \tilde{Y}]_{ \pm}\right]_{ \pm}=0
$$

if the $\sigma$ satisfies the following condition [19]:

$$
\sigma(a, b+c) \sigma^{-1}(a, b) \sigma^{-1}(a, c) \text { is invariant under cyclic permutation, } \forall a, b, c \in \Gamma \text {. }
$$

It turns out that (2.7) and (2.9) are equivalent to (2.6). This means that the algebra $\tilde{\mathfrak{g}}$ inherits the structure of a $\Gamma$-graded Lie (super)algebra.

In [19], a more general result was established, and a close relationship between $\Gamma$-graded Lie (super)algebras corresponding to different multiplication factors was established. In fact we can even (composing the Grassmann-hull and the results of Proposition 2.8) associate a $\Gamma$-graded-Lie algebra to a color Lie (super)algebra. This decoloration theorem was established in [1]. Let us briefly recall the main steps of its proof.

Consider $\mathfrak{g}=\underset{a \in \Gamma}{\oplus} \mathfrak{g}_{a}$ a color Lie (super)algebra with commutation factor $N$. Introduce also $\Lambda=\underset{a \in \Gamma}{\oplus} \Lambda_{a}$ a $\Gamma$-graded algebra, canonically generated by the variables $\theta_{i}^{a}, a \in \Gamma, i=1, \cdots, m_{a}$ satisfying

$$
\theta_{i}^{a} \theta_{j}^{b}-N^{-1}(a, b) \theta_{j}^{b} \theta_{i}^{a}=0 .
$$

Then the zero-graded part of $\mathfrak{g}(\Lambda)=\Lambda \otimes \mathfrak{g}$,

$$
\mathfrak{g}(\Lambda)_{0}=\underset{a \in \Gamma}{\oplus} \Lambda_{-a} \otimes \mathfrak{g}_{a}
$$

is a Lie algebra. Indeed, for $X, Y, Z \in \mathfrak{g}_{a} \cdot \mathfrak{g}_{b}, \mathfrak{g}_{c}$ and $\theta, \psi, \eta \in \Lambda_{-a}, \Lambda_{-b}, \Lambda_{-c}$, it is not difficult to check that following relations are satisfied:

$$
\begin{array}{ll}
\text { 1. } & {[\theta \otimes X, \psi \otimes Y]=\theta \psi \otimes[|X, Y|]_{N} \in \Lambda_{-a-b} \otimes \mathfrak{g}_{a+b}} \\
\text { 2. } & {[\theta \otimes X, \psi \otimes Y]=-[\psi \otimes Y, \theta \otimes X]} \\
\text { 3. } & {[\theta \otimes X,[\psi \otimes Y, \eta \otimes Z]]+[\psi \otimes Y,[\eta \otimes Z, \theta \otimes X]]+[\eta \otimes Z,[\theta \otimes X, \psi \otimes Y]]=0 .}
\end{array}
$$

This decoloration theorem has an interesting consequence. Specifically, it means that one can associate a group to a color Lie (super)algebra and that the parameters of the transformation are related to the algebra $\Lambda$ above. This result was used in the papers of Wills-Toro et al in the trefoil symmetry frame [13]. Finally, let us mention that this decoloration theorem is in some sense the inverse procedure to the one given in Example 2.7. 


\section{$3 \quad$ Lie algebras of order $F$}

Lie algebras of order $F$, introduced in [9], correspond to a different kind of extensions of Lie (super)algebras, motivated by the implementation of non-trivial extensions of the Poincaré algebra in QFT. This type of algebras is characterized by an hybrid multiplication law: part of the algebra is realized by a binary multiplication, while another part of the algebra is realized via an $F$-order product. More precisely, a Lie algebra of order $F$ is graded by the abelian group $\Gamma=\mathbb{Z}_{F}$. The zero-graded part is a Lie algebra and an $F$-fold symmetric product (playing the role of the anticommutator in the case $F=2$ ) expresses the zero graded part in terms of the non-zero graded part.

Definition 3.1 Let $F \in \mathbb{N}^{*}$. A $\mathbb{Z}_{F}$-graded $\mathbb{C}$-vector space $\mathfrak{g}=\mathfrak{g}_{0} \oplus \mathfrak{g}_{1} \oplus \mathfrak{g}_{2} \cdots \oplus \mathfrak{g}_{F-1}$ is called a complex Lie algebra of order $F$ if

1. $\mathfrak{g}_{0}$ is a complex Lie algebra.

2. For all $i=1, \ldots, F-1, \mathfrak{g}_{i}$ is a representation of $\mathfrak{g}_{0}$. If $X \in \mathfrak{g}_{0}, Y \in \mathfrak{g}_{i}$ then $[X, Y]$ denotes the action of $X$ on $Y$ for any $i=1, \cdots, F-1$.

3. For all $i=1, \ldots, F-1$ there exists an $F$-linear, $\mathfrak{g}_{0}$-equivariant map $\{\cdots\}: \mathcal{S}^{F}\left(\mathfrak{g}_{i}\right) \rightarrow \mathfrak{g}_{0}$, where $\mathcal{S}^{F}\left(\mathfrak{g}_{i}\right)$ denotes the $F$-fold symmetric product of $\mathfrak{g}_{i}$.

4. For all $X_{i} \in \mathfrak{g}_{0}$ and $Y_{j} \in \mathfrak{g}_{k}$ the following "Jacobi identities" hold:

$$
\begin{aligned}
& {\left[\left[X_{1}, X_{2}\right], X_{3}\right]+\left[\left[X_{2}, X_{3}\right], X_{1}\right]+\left[\left[X_{3}, X_{1}\right], X_{2}\right]=0,} \\
& {\left[\left[X_{1}, X_{2}\right], Y_{3}\right]+\left[\left[X_{2}, Y_{3}\right], X_{1}\right]+\left[\left[Y_{3}, X_{1}\right], X_{2}\right]=0,} \\
& {\left[X,\left\{Y_{1}, \ldots, Y_{F}\right\}\right]=\left\{\left[X, Y_{1}\right], \ldots, Y_{F}\right\}+\cdots+\left\{Y_{1}, \ldots,\left[X, Y_{F}\right]\right\}} \\
& \sum_{i=1}^{F+1}\left[Y_{i},\left\{Y_{1}, \ldots, Y_{i-1}, Y_{i+1}, \ldots, Y_{F+1}\right\}\right]=0
\end{aligned}
$$

Remark 3.2 If $F=1$, by definition $\mathfrak{g}=\mathfrak{g}_{0}$ and a Lie algebra of order 1 is a Lie algebra. If $F=2$, then $\mathfrak{g}$ is a Lie superalgebra. Therefore, Lie algebras of order $F$ appear as some kind of generalizations of Lie algebras and superalgebras.

Proposition 3.3 Let $\mathfrak{g}=\mathfrak{g}_{0} \oplus \mathfrak{g}_{1} \oplus \cdots \oplus \mathfrak{g}_{F-1}$ be a Lie algebra of order $F$, with $F>1$. For any $i=$ $1, \ldots, F-1$, the $\mathbb{Z}_{F}$-graded vector spaces $\mathfrak{g}_{0} \oplus \mathfrak{g}_{i}$ is a Lie algebra of order $F$. We call these type of algebras elementary Lie algebras of order $F$.

Remark 3.4 Let $\mathcal{A}=\mathcal{A}_{0} \oplus \mathcal{A}_{1} \oplus \cdots \oplus \mathcal{A}_{F-1}$ be an associative $\mathbb{Z}_{F}$-graded algebra with multiplication $\mu$. One can associate a Lie algebra of order $F$ to $\mathcal{A}$ as follows. For any $a_{0}, a_{0}^{\prime} \in \mathcal{A}_{0}, a_{1}, a_{2}, \cdots, a_{F} \in \mathcal{A}_{i}, i=$ $1, \cdots F-1$ we have

$$
\begin{aligned}
{\left[a_{0}, a_{0}^{\prime}\right] } & =\mu\left(a_{0}, a_{0}^{\prime}\right)-\mu\left(a_{0}^{\prime}, a_{0}\right) \in \mathcal{A}_{0}, \\
{\left[a_{0}, a_{1}\right] } & =\mu\left(a_{0}, a_{1}\right)-\mu\left(a_{1}, a_{0}\right) \in \mathcal{A}_{i}, \\
\left\{a_{1}, a_{2}, \cdots, a_{F}\right\} & =\mu\left(a_{1}, a_{2}, \cdots, a_{F}\right)+\text { perm. } \in \mathcal{A}_{0} .
\end{aligned}
$$

Furthermore, one can easily see that the Jacobi identities are a consequence of the associativity of the product $\mu$. Moreover, if $\mathcal{A}$ is an associative algebra and $\mathcal{C}_{F}^{1}$ the commutative $F$-dimensional algebra generated by a primitive element $e$ such that $e^{F}=1$, the algebra $\mathcal{C}_{F}^{1} \otimes \mathcal{A}=(1 \otimes \mathcal{A}) \oplus(e \otimes \mathcal{A}) \oplus \cdots \oplus\left(e^{F-1} \otimes \mathcal{A}\right)$ is $\mathbb{Z}_{F}$-graded, and thus leads to a Lie algebra of order $F$.

Definition 3.5 A representation of an elementary Lie algebra of order $F$ is a linear map $\rho: \mathfrak{g}=\mathfrak{g}_{0} \oplus \mathfrak{g}_{1} \rightarrow$ $\operatorname{End}(V)$, such that for all $X_{i} \in \mathfrak{g}_{0}, Y_{j} \in \mathfrak{g}_{1}$ 


$$
\begin{aligned}
& \rho\left(\left[X_{1}, X_{2}\right]\right)=\rho\left(X_{1}\right) \rho\left(X_{2}\right)-\rho\left(X_{2}\right) \rho\left(X_{1}\right), \\
& \rho\left(\left[X_{1}, Y_{2}\right]\right)=\rho\left(X_{1}\right) \rho\left(Y_{2}\right)-\rho\left(Y_{2}\right) \rho\left(X_{1}\right), \\
& \rho\left\{Y_{1}, \cdots, Y_{F}\right\}=\sum_{\sigma \in S_{F}} \rho\left(Y_{\sigma(1)}\right) \cdots \rho\left(Y_{\sigma(F)}\right) .
\end{aligned}
$$

$S_{F}$ being the symmetric group of $F$ elements.

By construction, the vector space $V$ is graded $V=V_{0} \oplus \cdots \oplus V_{F-1}$, and for all $a=\{0, \cdots, F-1\}, V_{a}$ is a $\mathfrak{g}_{0}$-module. Further, the condition $\rho\left(\mathfrak{g}_{1}\right)\left(V_{a}\right) \subseteq V_{a+1}$ holds.

Theorem 3.6 (M. Rausch de Traubenberg, M. J. Slupinski, [9]).

Let $\mathfrak{g}_{0}$ be a Lie algebra and $\mathfrak{g}_{1}$ be a $\mathfrak{g}_{0}$-module such that:

(i) $\mathfrak{g}=\mathfrak{g}_{0} \oplus \mathfrak{g}_{1}$ is a Lie algebra of order $F_{1}>1$;

(ii) $\mathfrak{g}_{1}$ admits a $\mathfrak{g}_{0}$-equivariant symmetric form of order $F_{2} \geq 1$.

Then $\mathfrak{g}=\mathfrak{g}_{0} \oplus \mathfrak{g}_{1}$ inherits the structure of a Lie algebra of order $F_{1}+F_{2}$.

The theorem above can be generalized to include the case $F_{1}=1$ [9].

Example 3.7 (This is a consequence of Theorem 3.6, modified to include $F_{1}=1$.) Let $\mathfrak{g}_{0}$ be any Lie algebra and let $\mathfrak{g}_{1}$ be its adjoint representation. Introduce $\left\{J_{a}, a=1, \cdots, \operatorname{dim} \mathfrak{g}_{0}\right\}$ a basis of $\mathfrak{g}_{0},\left\{A_{a}, a=\right.$ $\left.1, \cdots, \operatorname{dim} \mathfrak{g}_{0}\right\}$ the corresponding basis of $\mathfrak{g}_{1}$ and $g_{a b}=\operatorname{Tr}\left(A_{a} A_{b}\right)$ the Killing form. Then one can endow $\mathfrak{g}=\mathfrak{g}_{0} \oplus \mathfrak{g}_{1}$ with a Lie algebra of order 3 structure given by

$$
\begin{aligned}
{\left[J_{a}, J_{b}\right] } & =f_{a b}{ }^{c} J_{c}, \\
{\left[J_{a}, A_{b}\right] } & =f_{a b}{ }^{c} A_{c}, \\
\left\{A_{a}, A_{b}, A_{c}\right\} & =g_{a b} J_{c}+g_{a c} J_{b}+g_{b c} J_{a} .
\end{aligned}
$$

Example 3.8 Let $\mathfrak{g}_{0}=\left\langle L_{\mu \nu}=-L_{\nu \mu}, P_{\mu}, \mu, \nu=0, \cdots, D-1\right\rangle$ be the Poincaré algebra in $D$-dimensions and $\mathfrak{g}_{1}=\left\langle V_{\mu}, \mu=0, \cdots, D-1\right\rangle$ be the $D$-dimensional vector representation of $\mathfrak{g}_{0}$. The brackets

$$
\begin{aligned}
{\left[L_{\mu \nu}, L_{\rho \sigma}\right] } & =\eta_{\nu \sigma} L_{\rho \mu}-\eta_{\mu \sigma} L_{\rho \nu}+\eta_{\nu \rho} L_{\mu \sigma}-\eta_{\mu \rho} L_{\nu \sigma}, \\
{\left[L_{\mu \nu}, P_{\rho}\right] } & =\eta_{\nu \rho} P_{\mu}-\eta_{\mu \rho} P_{\nu},\left[L_{\mu \nu}, V_{\rho}\right]=\eta_{\nu \rho} V_{\mu}-\eta_{\mu \rho} V_{\nu},\left[P_{\mu}, V_{\nu}\right]=0, \\
\left\{V_{\mu}, V_{\nu}, V_{\rho}\right\} & =\eta_{\mu \nu} P_{\rho}+\eta_{\mu \rho} P_{\nu}+\eta_{\rho \nu} P_{\mu},
\end{aligned}
$$

with the metric $\eta_{\mu \nu}=\operatorname{diag}(1,-1, \cdots,-1)$ endow $\mathfrak{g}=\mathfrak{g}_{0} \oplus \mathfrak{g}_{1}$ with an elementary Lie algebra of order 3 structure which is denoted $I \mathfrak{s o}_{3}(1, D-1)$.

Example 3.9 Let mat $\left(m_{1}, m_{2}, m_{3}\right)$ and matel $\left(m_{1}, m_{2}, m_{3}\right)$ be the set of $\left(m_{1}+m_{2}+m_{3}\right) \times\left(m_{1}+m_{2}+m_{3}\right)$ matrices of the form

$$
\operatorname{mat}_{e l}\left(m_{1}, m_{2}, m_{3}\right)=\left\{\left(\begin{array}{ccc}
a_{0} & b_{1} & 0 \\
0 & a_{1} & b_{2} \\
b_{0} & 0 & a_{2}
\end{array}\right)\right\}, \quad \operatorname{mat}\left(m_{1}, m_{2}, m_{3}\right)=\left\{\left(\begin{array}{ccc}
a_{0} & b_{1} & c_{2} \\
c_{0} & a_{1} & b_{2} \\
b_{0} & c_{1} & a_{2}
\end{array}\right)\right\},
$$

with $a_{0} \in \mathfrak{g l}\left(m_{1}\right), a_{1} \in \mathfrak{g l}\left(m_{2}\right), a_{3} \in \mathfrak{g l}\left(m_{3}\right), b_{1} \in \mathcal{M}_{m_{1}, m_{2}}(\mathbb{C}), b_{2} \in \mathcal{M}_{m_{2}, m_{3}}(\mathbb{C}), b_{0} \in \mathcal{M}_{m_{3}, m_{1}}(\mathbb{C})$, and $c_{0} \in \mathcal{M}_{m_{2}, m_{1}}(\mathbb{C}), c_{1} \in \mathcal{M}_{m_{3}, m_{2}}(\mathbb{C}), c_{2} \in \mathcal{M}_{m_{1}, m_{3}}(\mathbb{C})$. A basis of this set of matrices can be constructed as follows. Consider the $\left(m_{1}+m_{2}+m_{3}\right)^{2}$ canonical matrices $e_{I}^{J}, 1 \leq I, J \leq m_{1}+m_{2}+m_{3}$. With the following convention for the indices $1 \leq i, j \leq m_{1}, m_{1}+1 \leq i^{\prime}, j^{\prime} \leq m_{1}+m_{2}, m_{1}+m_{2}+1 \leq i^{\prime \prime}, j^{\prime \prime} \leq m_{1}+m_{2}+m_{3}$, the generators are given by

$$
\begin{aligned}
& e_{i}^{j} \text { for } \mathfrak{g l}\left(m_{1}\right), \quad e_{i^{\prime}} j^{\prime} \text { for } \mathfrak{g l}\left(m_{2}\right), \quad e_{i^{\prime \prime}} j^{\prime \prime} \text { for } \mathfrak{g l}\left(m_{3}\right), \\
& e_{i}^{j^{\prime}} \text { for } \mathcal{M}_{m_{1}, m_{2}}(\mathbb{C}), \quad e_{i^{\prime}} j^{\prime \prime} \text { for } \mathcal{M}_{m_{2}, m_{3}}(\mathbb{C}), \quad e_{i^{\prime \prime}}{ }^{j} \text { for } \mathcal{M}_{m_{3}, m_{1}}(\mathbb{C}) \text {, } \\
& e_{i^{\prime}}{ }^{j} \text { for } \mathcal{M}_{m_{2}, m_{1}}(\mathbb{C}), \quad e_{i^{\prime \prime}} j^{\prime} \text { for } \mathcal{M}_{m_{3}, m_{2}}(\mathbb{C}), \quad e_{i}^{j^{\prime \prime}} \text { for } \mathcal{M}_{m_{1}, m_{3}}(\mathbb{C}) \text {. }
\end{aligned}
$$


Writing $\operatorname{mat}\left(m_{1}, m_{2}, m_{3}\right)=\operatorname{mat}\left(m_{1}, m_{2}, m_{3}\right)_{0} \oplus \operatorname{mat}\left(m_{1}, m_{2}, m_{3}\right)_{1} \oplus \operatorname{mat}\left(m_{1}, m_{2}, m_{3}\right)_{2}$ and mat $\operatorname{mal}_{1}\left(m_{1}, m_{2}, m_{3}\right)=$ $\operatorname{mat}_{\mathrm{el}}\left(m_{1}, m_{2}, m_{3}\right)_{0} \oplus$ matel $_{\mathrm{e}}\left(m_{1}, m_{2}, m_{3}\right)_{1}$, we denote generically by $X_{I} J$ the canonical generators of degree zero, $Y_{I}^{J}$ the canonical generators of degree one, and $Z_{I}{ }^{J}$ those of degree two. With these conventions, the brackets read

$$
\begin{aligned}
{\left[X_{I}{ }^{J}, X_{K}{ }^{L}\right] } & =\delta^{J}{ }_{K} X_{I}{ }^{L}-\delta^{L}{ }_{I} X_{K}{ }^{J}, \\
{\left[X_{I}{ }^{J}, Y_{K}{ }^{L}\right] } & =\delta^{J}{ }_{K} Y_{I}{ }^{L}-\delta^{L}{ }_{I} Y_{K}{ }^{J} \\
{\left[X_{I}{ }^{J}, Z_{K}{ }^{L}\right] } & =\delta^{J}{ }_{K} Z_{I}{ }^{L}-\delta^{L}{ }_{I} Z_{K}{ }^{J}, \\
\left\{Y_{I}{ }^{J}, Y_{K}{ }^{L}, Y_{M}{ }^{N}\right\} & =\delta^{J}{ }_{K} \delta^{L}{ }_{M} X_{I}{ }^{N}+\delta^{N}{ }_{I} \delta^{J}{ }_{K} X_{M}{ }^{L}+\delta^{L}{ }_{M} \delta^{N}{ }_{I} X_{K}{ }^{J} \\
& +\delta^{J}{ }_{M} \delta^{N}{ }_{K} X_{I}{ }^{L}+\delta^{N}{ }_{K} \delta^{L}{ }_{I} X_{M}{ }^{J}+\delta^{L}{ }_{I} \delta^{J}{ }_{M} X_{K}{ }^{N}, \\
\left\{Z_{I}{ }^{J}, Z_{K}{ }^{L}, Z_{M}{ }^{N}\right\} & =\delta^{J}{ }_{K} \delta^{L}{ }_{M} X_{I}{ }^{N}+\delta^{N}{ }_{I} \delta^{J}{ }_{K} X_{M}{ }^{L}+\delta^{L}{ }_{M} \delta^{N}{ }_{I} X_{K}{ }^{J} \\
& +\delta^{J}{ }_{M} \delta^{N}{ }_{K} X_{I}{ }^{L}+\delta^{N}{ }_{K} \delta^{L}{ }_{I} X_{M}{ }^{J}+\delta^{L}{ }_{I}{ }^{J}{ }^{J}{ }_{M} X_{K}{ }^{N} .
\end{aligned}
$$

This shows that $\operatorname{mat}\left(m_{1}, m_{2}, m_{3}\right)$ (resp. mat $\left.{ }_{\mathrm{el}}\left(m_{1}, m_{2}, m_{3}\right)\right)$ is endowed with the structure of Lie algebra of order three (resp. a structure of an elementary Lie algebra of order three). In particular, when $m_{1}=m_{2}=m_{3}$ the algebra above can be rewritten as $\operatorname{mat}(m, m, m)=\mathcal{C}_{3}^{1} \otimes \mathfrak{g l}(m)$, with $e=\left(\begin{array}{ccc}0 & 1 & 0 \\ 0 & 0 & 1 \\ 1 & 0 & 0\end{array}\right)$ being a faithful matrix representation of the canonical generator of $\mathcal{C}_{n}^{1}$.

The question to find appropriate variables to represent Lie algebras of order $F$ is much more involved than for color algebras. However, in some specific cases, we were able to find appropriate variables (see N. Mohammedi, G. Moultaka and M. Rausch de Traubenberg in [14]), and it turns out that these variables are strongly related to Clifford algebras of polynomials [18]. We will give another realization below.

\section{Color Lie algebras of order $F$}

Color Lie (super)algebras of order $F$ can be seen as a synthesis of the two types of algebras introduced previously. Indeed, for such algebras, we have simultaneously a binary product associated with a commutation factor and an $F$-order product. The latter is no more fully symmetric, but is also associated with the commutation factor. In this section we focus on color Lie (super)algebra of order 3.

Definition 4.1 Let $\Gamma$ be an abelian group and $N$ be a commutation factor, $\mathfrak{g}=\mathfrak{g}_{0} \oplus \mathfrak{g}_{1}$ is an elementary color Lie (super)algebra of order 3 if

1. $\mathfrak{g}_{0}=\underset{a \in \Gamma}{\oplus} \mathfrak{g}_{0, a}$ is a color Lie (super)algebra.

2. $\mathfrak{g}_{1}=\underset{a \in \Gamma}{\oplus} \mathfrak{g}_{1, a}$ is a representation of $\mathfrak{g}_{0}$. If $X \in \mathfrak{g}_{0}, Y \in \mathfrak{g}_{1}$ are homogeneous elements, then $[|X, Y|]_{N}$ denotes the action of $X$ on $Y$.

3. There exists a $\mathfrak{g}_{0}$-equivariant map $\{|., . .,|\}_{N}: \mathfrak{g}_{1} \otimes \mathfrak{g}_{1} \otimes \mathfrak{g}_{1} \rightarrow \mathfrak{g}_{0}$ such that for all $Y_{1} \in \mathfrak{g}_{1, a}, Y_{2} \in$ $\mathfrak{g}_{1, b}, Y_{3} \in \mathfrak{g}_{1, c}$ we have $\left\{\left|Y_{1}, Y_{2}, Y_{3}\right|\right\}_{N}=N(a, b)\left\{\left|Y_{2}, Y_{1}, Y_{3}\right|\right\}_{N}=N(b, c)\left\{\left|Y_{a}, Y_{c}, Y_{b}\right|\right\}_{N}$.

4. The following "Jacobi identities" hold: 


$$
\begin{aligned}
& {\left[\left|X_{1},\left[\left|X_{2}, X_{3}\right|\right]_{N}\right|\right]_{N}=\left[\left|\left[\left|X_{1}, X_{2}\right|\right]_{N}, X_{3}\right|\right]_{N}+N(a, b)\left[\left|X_{2},\left[\left|X_{1}, X_{3}\right|\right]_{N}\right|\right]_{N},} \\
& \forall\left(X_{1}, X_{2}, X_{3}\right) \in \mathfrak{g}_{0, a} \times \mathfrak{g}_{0, b} \times \mathfrak{g}_{0, c}, \\
& {\left[\left|X_{1},\left[\left|X_{2}, Y_{3}\right|\right]_{N}\right|\right]_{N}=\left[\left|\left[\left|X_{1}, X_{2}\right|\right]_{N}, Y_{3}\right|\right]_{N}+N(a, b)\left[\left|X_{2},\left[\left|X_{1}, Y_{3}\right|\right]_{N}\right|\right]_{N},} \\
& \forall X_{1} \in \mathfrak{g}_{0, a}, X_{2} \in \mathfrak{g}_{0, b}, Y_{3} \in \mathfrak{g}_{1, c}, \\
& {\left[\left|X,\left\{\left|Y_{1}, Y_{2}, Y_{3}\right|\right\}_{N}\right|\right]_{N}=\left\{\left|\left[\left|X_{1}, Y_{1}\right|\right]_{N}, Y_{2}, Y_{3}\right|\right\}_{N},} \\
& +N(a, b)\left\{\left|Y_{1},\left[\left|X_{1}, Y_{2}\right|\right]_{N}, Y_{3}\right|\right\}_{N}+N(a, b+c)\left\{\left|Y_{1}, Y_{2},\left[\left|X_{1}, Y_{3}\right|\right]_{N}\right|\right\}_{N}, \\
& \forall X \in \mathfrak{g}_{0, a}, Y_{1} \in \mathfrak{g}_{1, b}, Y_{2} \in \mathfrak{g}_{1, c}, Y_{3} \in \mathfrak{g}_{1, d} \\
& \left.0=\left[\left|Y_{1},\left\{\left|Y_{2}, Y_{3}, Y_{4}\right|\right\}_{N}\right|\right]\right]_{N}+N(a, b+c+d)\left[\left|Y_{2},\left\{\left|Y_{3}, Y_{4}, Y_{1}\right|\right]_{N}\right|\right\}_{N}+ \\
& N(a, b+c+d) N(b, a+c+d)\left[\left|Y_{3},\left\{\left|Y_{4}, Y_{1}, Y_{2}\right|\right\}_{N}\right|\right]_{N}+ \\
& N(a, b+c+d) N(b, a+c+d) N(c, a+b+d)\left[\left|Y_{4},\left\{\left|Y_{1}, Y_{2}, Y_{3}\right|\right\}_{N}\right|\right]_{N}, \\
& \forall Y_{1} \in \mathfrak{g}_{1, a}, Y_{2} \in \mathfrak{g}_{1, b}, Y_{3} \in \mathfrak{g}_{1, c}, Y_{4} \in \mathfrak{g}_{1, d}
\end{aligned}
$$

We observe that if $\Gamma=\Gamma_{0}+\Gamma_{1}$ is a decomposition of $\Gamma$ with respect to its $\mathbb{Z}_{2}$-grading, as seen in Section 2 , and such that $\Gamma_{1}=0$ (resp. $\Gamma_{1} \neq 0$ ), then $\mathfrak{g}$ is called a color Lie algebra (resp. superalgebra). Moreover, if $\Gamma=\mathbb{Z}_{2}$ and $N(1,1)=-1$ hold, the algebra $\mathfrak{g}$ is called a Lie superalgebra of order three.

Definition 4.2 A representation of an elementary color Lie (super)algebra of order 3 is a linear map $\rho$ : $\mathfrak{g} \rightarrow \operatorname{End}(V)$ satisfying the conditions

1. $\rho\left(\left[\left|X_{1}, X_{2}\right|\right]_{N}\right)=\rho\left(X_{1}\right) \rho\left(X_{2}\right)-N(a, b) \rho\left(X_{2}\right) \rho\left(X_{1}\right)$, for all $X_{1} \in \mathfrak{g}_{0, a}, X_{2} \in \mathfrak{g}_{0, b}$;

2. $\rho\left(\left[\left|X_{1}, Y_{2}\right|\right]_{N}\right)=\rho\left(X_{1}\right) \rho\left(Y_{2}\right)-N(a, b) \rho\left(Y_{2}\right) \rho\left(X_{1}\right)$, for all $X_{1} \in \mathfrak{g}_{0, a}, Y_{2} \in \mathfrak{g}_{1, b}$;

3. $\rho\left(\left\{\left|Y_{1}, Y_{2}, Y_{3}\right|\right\}_{N}\right)=\rho\left(Y_{1}\right) \rho\left(Y_{2}\right) \rho\left(Y_{3}\right)+N(a, b) N(a, c) \rho\left(Y_{2}\right) \rho\left(Y_{3}\right) \rho\left(Y_{1}\right)+N(b, c) N(a, c) \rho\left(Y_{3}\right) \rho\left(Y_{1}\right) \rho\left(Y_{2}\right)+$ $N(b, c) \rho\left(Y_{1}\right) \rho\left(Y_{3}\right) \rho\left(Y_{2}\right)+N(a, b) \rho\left(Y_{2}\right) \rho\left(Y_{1}\right) \rho\left(Y_{3}\right)+N(a, b) N(a, c) N(b, c) \rho\left(Y_{3}\right) \rho\left(Y_{2}\right) \rho\left(Y_{1}\right)$, for all $Y_{1} \in$ $\mathfrak{g}_{1, a}, Y_{2} \in \mathfrak{g}_{1, b}, Y_{3} \in \mathfrak{g}_{1, c}$.

By construction, the vector space $V$ is graded and we have $V=V_{0} \oplus V_{1} \oplus V_{2}$ with $V_{i}=\underset{a \in \Gamma}{\oplus} V_{i, a}$. Furthermore, each $V_{i, a}$ is a $\mathfrak{g}_{0,0}$-module and the inclusion relation $\rho\left(\mathfrak{g}_{i, a}\right) V_{j, b} \subseteq V_{i+j, a+b}$ holds.

Remark 4.3 Let $\left.\mathcal{A}=\mathcal{A}_{0} \oplus \mathcal{A}_{1} \oplus \mathcal{A}_{2}=\left(\underset{a \in \Gamma}{\oplus} \mathcal{A}_{0, a}\right) \oplus\left(\underset{a \in \Gamma}{\oplus} \mathcal{A}_{1, a}\right) \oplus \underset{a \in \Gamma}{\oplus} \mathcal{A}_{2, a}\right)$ be an associative $\mathbb{Z}_{3} \times \Gamma$-graded algebra with multiplication $\mu$. One can associate a color Lie superalgebra of order three to $\mathcal{A}$ defining the products in a similar manner as in Remarks 2.4 and 3.4. In this case, the Jacobi identities are also a consequence of the associativity of the product $\mu$. Similarly, if $\mathcal{A}$ is an associative $\Gamma$-algebra and $\mathcal{C}_{3}^{1}$ the commutative three-dimensional algebra generated by a primitive element $e$ such that $e^{3}=1$, the algebra $\mathcal{C}_{3}^{1} \otimes \mathcal{A}=(1 \otimes \mathcal{A}) \oplus(e \otimes \mathcal{A}) \oplus\left(e^{2} \otimes \mathcal{A}\right)$ is associative and $\mathbb{Z}_{3} \times \Gamma$-graded, and therefore leads to a color Lie algebra of order three.

The examples of color Lie (super)algebras of order $F$ are basically of two types: we can construct a color Lie (super)algebra of order $F$ from either a color Lie (super)algebra or a Lie algebra of order $F$.

Example 4.4 Let $\mathfrak{g r}\left(\{m\}_{\Gamma, N}\right)=\underset{a \in \Gamma}{\oplus} \mathfrak{g}_{a}$ be the color Lie (super)algebra of Example 2.6 and let $\mathcal{C}_{3}^{1}$ be the generalised Clifford algebra with canonical generator $e$, then

1. $\mathcal{C}_{3}^{1} \otimes \mathfrak{g}$ is a color Lie (super)algebra of order 3 ;

2. $\langle 1, e\rangle \otimes \mathfrak{g}$ is an elementary color Lie (super)algebra of order 3 .

For the second algebra, following the notations of Example 2.6 we denote $E^{p}{ }_{q}$ a basis of $\mathfrak{g l}\left(\{m\}_{\Gamma, N}\right)$ and $X^{p}{ }_{q}=\mathbf{1} \otimes E^{p}{ }_{q}\left(\right.$ resp. $\left.Y^{p}{ }_{q}=e \otimes E^{p}{ }_{q}\right)$ a basis of $\mathbf{1} \otimes \mathfrak{g l}\left(\{m\}_{\Gamma, N}\right)$ (resp. of $\left.e \otimes \mathfrak{g l}\left(\{m\}_{\Gamma, N}\right)\right)$. Then, the trilinear brackets read 


$$
\begin{aligned}
& \left\{\left|Y^{p}{ }_{q}, Y^{r}{ }_{s}, Y^{t}{ }_{u}\right|\right\}_{N}=\delta_{q}{ }^{r} \delta_{s}{ }^{t} X^{p}{ }_{u}+ \\
& +N(\operatorname{gr}(q)-\operatorname{gr}(p), \operatorname{gr}(s)-\operatorname{gr}(r)) N(\operatorname{gr}(q)-\operatorname{gr}(p), \operatorname{gr}(u)-\operatorname{gr}(t)) \delta_{s}{ }^{t} \delta_{u}{ }^{p} X^{r}{ }_{q} \\
& +N(\operatorname{gr}(q)-\operatorname{gr}(p), \operatorname{gr}(u)-\operatorname{gr}(t)) N(\operatorname{gr}(s)-\operatorname{gr}(r), \operatorname{gr}(u)-\operatorname{gr}(t)) \delta_{u}{ }^{p} \delta_{q}{ }^{r} X^{t}{ }_{s} \\
& +N(\operatorname{gr}(s)-\operatorname{gr}(r), \operatorname{gr}(u)-\operatorname{gr}(t)) \delta_{q}{ }^{t} \delta_{u}{ }^{r} X^{p}{ }_{s}+N(\operatorname{gr}(q)-\operatorname{gr}(p), \operatorname{gr}(s)-\operatorname{gr}(r)) \delta_{s}{ }^{p} \delta_{q}{ }^{t} X^{r}{ }_{u} \\
& +N(\operatorname{gr}(q)-\operatorname{gr}(p), \operatorname{gr}(s)-\operatorname{gr}(r)) N(\operatorname{gr}(q)-\operatorname{gr}(p), \operatorname{gr}(u)-\operatorname{gr}(t)) N(\operatorname{gr}(s)-\operatorname{gr}(r), \operatorname{gr}(u)-\operatorname{gr}(t)) \delta_{u}{ }^{r} \delta_{s}{ }^{p} X^{t}{ }_{q} .
\end{aligned}
$$

Example 4.5 Let $\mathfrak{g}$ be an arbitrary (elementary) Lie algebra of order 3 and let $\mathcal{C}_{n}^{2}$ be the generalized Clifford algebra with canonical generators $e_{1}, e_{2}$, then $\mathcal{C}_{n}^{2} \otimes \mathfrak{g}$ is a color Lie algebra of order 3 with abelian group $\mathbb{Z}_{n} \times \mathbb{Z}_{n}$ and commutation factor $N\left(\left(a_{1}, b_{1}\right),\left(a_{2}, b_{2}\right)\right)=q^{a_{1} b_{2}-a_{2} b_{1}}$. Suppose that an elementary Lie algebra of order $3 \mathfrak{g}=\mathfrak{g}_{0} \oplus \mathfrak{g}_{1}$ is given. Denote $\left\{X_{\alpha}, \alpha=1, \cdots, \operatorname{dim}\left(\mathfrak{g}_{0}\right)\right\}\left(\operatorname{resp} .\left\{Y_{m}, m=1, \cdots, \operatorname{dim}\left(\mathfrak{g}_{1}\right)\right\}\right)$ a basis of $\mathfrak{g}_{0}\left(\right.$ resp. $\mathfrak{g}_{1}$.) such that

$$
\left[X_{\alpha}, X_{\beta}\right]=f_{\alpha \beta}{ }^{\gamma} X_{\beta},\left[X_{\alpha}, Y_{m}\right]=R_{\alpha m}{ }^{n} Y_{n},\left\{Y_{m}, Y_{n}, Y_{p}\right\}=Q_{m n p}{ }^{\alpha} X_{\alpha} .
$$

Define $\mathfrak{g}_{0,(a, b)}=\left\{X_{\alpha}{ }^{(a, b)}=\rho_{(a, b)} \otimes X^{\alpha}\right\}$ and $\mathfrak{g}_{1,(a, b)}=\left\{Y_{m}^{(a, b)}=\rho_{(a, b)} \otimes Y^{m}\right\}$ (with $\rho_{(a, b)}=e_{1}^{a} e_{2}^{b}$ ), we thus have $\underset{(a, b) \in \mathbb{Z}_{n} \times \mathbb{Z}_{n}}{\mathfrak{g}_{0,(a, b)}} \bigoplus_{(a, b) \in \mathbb{Z}_{n} \times \mathbb{Z}_{n}} \mathfrak{g}_{1,(a, b)}$ is an elementary color Lie algebra of order 3 with brackets:

$$
\begin{aligned}
{\left[\left|X_{\alpha}^{(a, b)}, X_{\beta}^{(c, d)}\right|\right]_{N} } & =q^{-b c} f_{\alpha \beta}{ }^{\gamma} X_{\beta}^{(a+c, b+d)}, \\
{\left[\left|X_{\alpha}^{(a, b)}, Y_{m}^{(c, d)}\right|\right]_{N} } & =q^{-b c} R_{\alpha m}{ }^{n} Y_{n}^{(a+c, b+d)}, \\
\left\{\left|Y_{m}^{(a, b)}, Y_{n}^{(c, d)}, Y_{p}^{(e, f)}\right|\right\}_{N} & =q^{-b(c+e)-d e} Q_{m n p}{ }^{\alpha} X_{\alpha}^{(a+c+e, b+d+f)} .
\end{aligned}
$$

As in Example 2.7 this can be extended for $\Gamma=\mathbb{Z}_{n}^{N}$ and for color Lie superalgebras of order three.

Example 4.6 This example is a synthesis of Examples 2.6 and 3.9. Consider three abelian groups $\Gamma_{1}, \Gamma_{2}, \Gamma_{3}$ and corresponding commutation factors $N_{1}, N_{2}, N_{3}$. Then we define on the group $\Gamma=\Gamma_{1} \times \Gamma_{2} \times \Gamma_{3}$ the commutation factor $N(\vec{a}, \vec{b})=N_{1}\left(a_{1}, b_{1}\right) N_{2}\left(a_{2}, b_{2}\right) N_{3}\left(a_{3}, b_{3}\right)$, with $\vec{a}=\left(a_{1}, a_{2}, a_{3}\right) \in \Gamma$ etc. Let $m_{i}=$ $m_{i, 1}+\cdots+m_{i, n_{i}}$ with $i=1,2,3$ be three integers and $\mathfrak{g l}\left(\left\{m_{1}\right\}_{\Gamma_{1}, N_{1}}\right), \mathfrak{g l}\left(\left\{m_{2}\right\}_{\Gamma_{2}, N_{2}}\right), \mathfrak{g l}\left(\left\{m_{3}\right\}_{\Gamma_{3}, N_{3}}\right)$ be three color Lie (super)algebras as in Example 2.6. Introduce now the matrices $\mathcal{M}_{m_{1}, m_{2}}(\mathbb{C})$ in the fundamental representation of $\mathfrak{g l}\left(\left\{m_{1}\right\}_{\Gamma_{1}, N_{1}}\right)$ and in the dual of the fundamental representation of $\mathfrak{g l}\left(\left\{m_{2}\right\}_{\Gamma_{2}, N_{2}}\right)$. In a similar way as in Example 3.9, we consider the set of matrices $\mathcal{M}_{m_{2}, m_{3}}(\mathbb{C})$ and $\mathcal{M}_{m_{3}, m_{1}}(\mathbb{C})$ and the algebra

$$
\mathfrak{g}=\left(\begin{array}{ccc}
\mathfrak{g l}\left(\left\{m_{1}\right\}_{\Gamma_{1}, N_{1}}\right) & \mathcal{M}_{m_{1}, m_{2}}(\mathbb{C}) & 0 \\
0 & \mathfrak{g l}\left(\left\{m_{2}\right\}_{\Gamma_{2}, N_{2}}\right) & \mathcal{M}_{m_{2}, m_{3}}(\mathbb{C}) \\
\mathcal{M}_{m_{3}, m_{1}}(\mathbb{C}) & 0 & \mathfrak{g l}\left(\left\{m_{3}\right\}_{\Gamma_{3}, N_{3}}\right)
\end{array}\right)=\left(\begin{array}{ccc}
X_{i^{j}}{ }^{j} & Y_{i}^{j^{\prime}} & 0 \\
0 & X_{i^{\prime}} j^{\prime} & Y_{i^{\prime}} j^{\prime \prime} \\
Y_{i^{\prime \prime}}{ }^{j} & 0 & X_{i^{\prime \prime}} j^{\prime \prime}
\end{array}\right)
$$

with the notations of Examples 3.9. It is obviously a color Lie (super)algebra of order three. The various brackets are similar to those of Example 3.9 and 2.6. We just give a few brackets for completeness:

$$
\begin{aligned}
& \begin{array}{ll}
{\left[\left|X_{i}{ }^{j}, Y_{k} \ell^{\ell^{\prime}}\right|\right]_{1}} & =\delta_{k}{ }^{j} Y_{i}^{\ell^{\prime}}, \\
{\left[\left|X_{i^{\prime}}{ }^{\prime}, Y_{k} \ell^{\prime}\right|\right]_{N}} & =-N_{2}\left(\operatorname{gr}\left(i^{\prime}\right)-\operatorname{gr}\left(j^{\prime}\right),-\operatorname{gr}\left(\ell^{\prime}\right)\right) \delta_{i^{\prime}} \ell^{\ell^{\prime}} Y_{k} j^{j^{\prime}},
\end{array} \\
& \left\{\left|Y_{i}^{j^{\prime}}, Y_{k^{\prime}}{ }^{\ell^{\prime \prime}}, Y_{m^{\prime \prime}} n\right|\right\}_{N}=\delta^{j^{\prime}}{ }_{k^{\prime}} \delta^{\ell^{\prime \prime}}{ }_{m^{\prime \prime}} X^{i}{ }_{n}+N_{2}\left(-\operatorname{gr}\left(j^{\prime}\right), \operatorname{gr}\left(k^{\prime}\right)\right) N_{1}(\operatorname{gr}(i),-\operatorname{gr}(n)) \delta^{\ell^{\prime \prime}}{ }_{m^{\prime \prime}} \delta^{n}{ }_{i} X_{k^{\prime}} j^{\prime} \\
& +N_{3}\left(-\operatorname{gr}\left(\ell^{\prime}\right), \operatorname{gr}\left(m^{\prime \prime}\right)\right) N_{1}(\operatorname{gr}(i),-\operatorname{gr}(n)) \delta_{i}^{n} \delta^{j^{\prime}}{ }_{k^{\prime}} X_{m^{\prime \prime}} \ell^{\prime \prime} \text {. }
\end{aligned}
$$


To conclude this section, we now show that there is an analogous of the decoloration theorem established in Section 2. As done there, one can proceed in two different (but related) ways. To set up the main result of this theorem, consider $\mathfrak{g}=\left(\underset{a \in \Gamma}{\oplus} \mathfrak{g}_{0, a}\right) \oplus\left(\underset{a \in \Gamma}{\oplus} \mathfrak{g}_{1, a}\right)$ a color Lie superalgebra with grading abelian group $\Gamma$ and commutation factor $N$. In the second approach we directly associate to $\mathfrak{g}$ a Lie algebra of order three, in the same manner as in Section 2 by considering the algebra $\Lambda=\underset{a \in \Gamma}{\Lambda_{a}}$, where $\Lambda_{a}$ is generated by the variables $\theta_{i}^{a}$ satisfying equation (2.10). The algebra

$$
\mathfrak{g}(\Lambda)_{0}=\left(\underset{a \in \Gamma}{\oplus} \Lambda_{-a} \otimes \mathfrak{g}_{0, a}\right) \oplus\left(\underset{a \in \Gamma}{\oplus} \Lambda_{-a} \otimes \mathfrak{g}_{1, a}\right)
$$

is a Lie algebra of order three. This is proved in a similar way as in Section 2 and only the trilinear bracket are slightly different. Let $X \in \mathfrak{g}_{a}, Y \in \mathfrak{g}_{b}, Z \in \mathfrak{g}_{c}$ and $\theta \in \Lambda_{-a}, \psi \in \Lambda_{-b}, \eta \in \Lambda_{-c}$. It is not difficult to check that

$$
\{\theta \otimes X, \psi \otimes Y, \eta \otimes Z\}=\theta \psi \eta \otimes\{|X, Y, Z|\}_{N} \in \Lambda_{-a-b-c} \otimes \mathfrak{g}_{0, a+b+c} .
$$

The Jacobi identities involving trilinear brackets are a consequence of the identity $\left[\theta_{1} \otimes Y_{1},\left\{\theta_{2} \otimes Y_{2}, \theta_{3} \otimes\right.\right.$ $\left.\left.Y_{3}, \theta_{4} \otimes Y_{4}\right\}\right]=\theta_{1} \theta_{2} \theta_{3} \theta_{4}\left[\left|Y_{1},\left\{\left|Y_{2}, Y_{3}, Y_{4}\right|\right\}_{N}\right|\right]_{N}$ (for any $\left.Y_{i} \in \mathfrak{g}_{1, a_{i}}, \theta_{i} \in \Lambda_{-a_{i}}, i=1, \cdots, 4\right)$ together with the associativity of the product in $\Lambda$ and equation (2.10). This proves that $\mathfrak{g}(\Lambda)_{0}$ is a Lie algebra of order three.

In the first correspondence we introduce $N_{+}$as in Section 2 and the variables $e_{a}$ as in Proposition 2.8 satisfying equations (2.5) and (2.6). Recall that the last property ensures that the product is associative. Then the algebra $\tilde{\mathfrak{g}}=\left(\underset{a \in \Gamma}{\oplus} e_{-a} \otimes \mathfrak{g}_{0, a}\right) \oplus\left(\underset{a \in \Gamma}{\oplus} e_{-a} \otimes \mathfrak{g}_{1, a}\right)$ is a Lie (super)algebra of order three. The proof goes along the same lines as in Proposition 2.8. For the bilinear part the proof is the same as in Proposition 2.8. For the cubic bracket, if we take $X \in \mathfrak{g}_{a}, Y \in \mathfrak{g}_{b}$ and $Z \in \mathfrak{g}_{a}$, a simple calculation shows, using condition (2.6), the explicit structure of the trilinear bracket:

$$
\begin{aligned}
& \left\{e_{-a} \otimes X, e_{-b} \otimes Y, e_{-c} \otimes Z\right\}_{ \pm}=\left(e_{-a} \otimes X\right)\left(e_{-b} \otimes Y\right)\left(e_{-c} \otimes Z\right)+ \\
& \quad(-1)^{|X|(|Y|+|Z|)}\left(e_{-b} \otimes Y\right)\left(e_{-c} \otimes Z\right)\left(e_{-a} \otimes X\right)+(-1)^{|Z|(|X|+|Y|)}\left(e_{-c} \otimes Z\right)\left(e_{-a} \otimes X\right)\left(e_{-b} \otimes Y\right) \\
& \quad+(-1)^{|Z||Y|}\left(e_{-a} \otimes X\right)\left(e_{-c} \otimes Z\right)\left(e_{-b} \otimes Y\right)+(-1)^{|Y||X|}\left(e_{-b} \otimes Y\right)\left(e_{-a} \otimes X\right)\left(e_{-c} \otimes Z\right) \\
& \quad+(-1)^{|X||Y|+|X||Z|+|Y||Z|}\left(e_{-c} \otimes Z\right)\left(e_{-b} \otimes Y\right)\left(e_{-a} \otimes X\right) \\
& \quad=\sigma(-a,-b) \sigma(-a-b,-c) e_{a+b+c} \otimes\{|X, Y, X|\}_{N} \in e_{-a-b-c} \otimes \mathfrak{g}_{0, a+b+c},
\end{aligned}
$$

where $|X|$ denotes the degree of $X$ with respect to the $\mathbb{Z}_{2}$ grading of $\Gamma=\Gamma_{0} \oplus \Gamma_{1}$ etc.. Since we have $e_{a} e_{b}-N_{+}^{-1}(a, b) e_{b} e_{a}=0$, and the algebra $G$ is associative, there is no need to prove the Jacobi identities involving trilinear brackets (the proof being the same as in previous cases). This illustrates how we can associate a Lie (super)algebra of order three to a color Lie (super)algebra of order three. These results, taken together, can be resumed in uniform manner in the following decoloration theorem:

Proposition 4.7 There is an isomorphism between color Lie (super)algebras of order three and Lie (super)algebras of order three.

This theorem can be seen as a Grassmann-hull that replaces a Lie superalgebra by a Lie algebra introducing Grassmann variables. It can be further be seen as a kind of Jordan-Wigner transformation in physical applications.

To finish this section let us observe the following. The decoloration theorem above and that of Section 2 seem to indicate that color Lie (super)algebras (resp. color Lie (super)algebras of order three) do not really constitute new objects, since they are isomorphic to Lie algebras (resp. Lie algebras of order three). In fact, as a consequence of these theorems, for any representation $\mathcal{R}$ of a color algebra $\mathfrak{g}$ we can construct, by means of the procedure above, an isomorphic representation of the associated non-color algebra. The converse of this procedure also holds. It should however taken into account that this property does not imply that all 
representations of color (resp. non-color) algebras are obtained from representations of the corresponding non-color (resp. color) algebras. ${ }^{1}$

\section{Quons and realization of color Lie (super)algebras of order 3}

Quons where conceived in particle statistics as one of the alternatives to construct theories were either the Bose or Fermi statistics are violated by a small amount [7]. Although observables related to particles subjected to this type of intermediate statistics fail to have the usual locality properties, their validity in nonrelativistic field theory and free field theories obeying the TCP theorem has been shown. In this section we prove that color Lie algebras of order 3 admitting a finite dimensional linear representation can be realized by means of quon algebras for the important case $q=0$. This result is a generalisation of various properties that are well known for the usual boson and fermion algebras.

Let $-1 \leq q \leq 1$ and consider the variables $a_{i}, a^{i}, i=1, \cdots n$. We define the $q$-mutator or quon-algebra by means of

$$
a_{i} a^{j}-q a^{j} a_{i}=\delta_{i}^{j}
$$

where no relation between variables of the same type are postulated. The (complex) quon algebra is denoted by $\mathcal{Q}_{n, q}(\mathbb{C}) .{ }^{2}$ For the two extreme values of $q$ we recover the well known statistics. If $q=-1$, together with the relations $a^{i} a^{j}+a^{j} a^{i}=0$ and $a_{i} a_{j}+a_{j} a_{i}=0$, the quon algebra reduces to the fermion algebra. For $q=1$, together with $a^{i} a^{j}-a^{j} a^{i}=0$ and $a_{i} a_{j}-a_{j} a_{i}=0$, it reproduces the boson algebra. Therefore, the quon algebra can be interpreted as an interpolation between Bose and Fermi statistics. ${ }^{3}$

Lemma 5.1 Let $M_{1}, \cdots, M_{k}$ be $(n \times n)$ complex matrices satisfying a polynomial relation $P\left(M_{1}, \cdots, M_{k}\right)=$ 0 . Then there exists $k$ elements $\mathcal{M}_{k} \in \mathcal{Q}_{n, 0}(\mathbb{C})(k=1, \cdots, n)$ such that $P\left(\mathcal{M}_{1}, \cdots, \mathcal{M}_{n}\right)=0$.

Proof. Given two arbitrary generators $a_{i}, a^{i}$ of $\mathcal{Q}_{n, 0}(\mathbb{C})$, by equation 5.1 we have $a_{i} a^{j}=\delta_{i}{ }^{j}$. This means in particular that the $n^{2}$ elements $e^{i}{ }_{j}$ defined by $e^{i}{ }_{j}=a^{i} a_{j}, 1 \leq i, j \leq n$ of $\mathcal{Q}_{n, 0}$ satisfy the relation $e^{i}{ }_{j} e^{k} \ell=\delta_{j}{ }^{k} e^{i} \ell$. Denoting by $E^{i}{ }_{j}$ the canonical generators of $\mathcal{M}_{n}(\mathbb{C})$ (the $(n \times n)$ complex matrices), the mapping $f: \mathcal{M}_{n}(\mathbb{C}) \rightarrow \mathcal{Q}_{n, o}$ defined by $f\left(E^{i}{ }_{j}\right)=e^{i}{ }_{j}$ turns out to be a injection. Therefore, since there is no kernel, the elements $\mathcal{M}_{k}=a^{i}\left(M_{k}\right)_{i}{ }^{j} a_{j} \in \mathcal{Q}_{n, 0}(\mathbb{C})$ have to satisfy the same relations as the matrices $M_{k}$. Thus $P\left(\mathcal{M}_{1}, \cdots, \mathcal{M}_{n}\right)=0$.

The quon algebra with $q=0$ has been studied in detail by Greenberg, and constitutes an example of "infinite statistics" [7]. It was moreover shown there that the $q=0$ operators can be used as building blocks for representations in the general $|q| \neq 1$ case. We next show that, under special circumstances, color Lie algebras of order 3 naturally embed into a $q=0$ quon algebra.

Theorem 5.2 Let $\Gamma$ be an abelian group, $N$ a commutation factor and $\mathfrak{g}$ be a color Lie (super)algebra of order 3. If $\mathfrak{g}$ admits a finite dimensional matrix representation, then $\mathfrak{g}$ can be realized by a quon algebra with $q=0$.

Proof. Suppose that the decomposition $\mathfrak{g}=\mathfrak{g}_{0} \oplus \mathfrak{g}_{1}=\underset{a \in \Gamma}{\oplus} \mathfrak{g}_{0, a} \underset{a \in \Gamma}{\oplus} \mathfrak{g}_{1, a}$ with respect to the abelian group $\Gamma$ is given. Let $X_{\alpha}^{(a)}$ be a basis of $\mathfrak{g}_{0, a}$ and $Y_{m}^{(a)}$ be a basis of $\mathfrak{g}_{1, a}$ such that the following relations hold:

$$
\begin{aligned}
& {\left[\left|X_{\alpha}{ }^{(a)}, X_{\beta}^{(b)}\right|\right]_{N}=C^{(a, b)}{ }_{\alpha \beta^{\gamma}} X_{\gamma}^{(a+b)}, \quad\left[\left|X_{\alpha}{ }^{(a)}, Y_{m}{ }^{(b)}\right|\right]_{N}=R^{(a, b)}{ }_{\alpha m}{ }^{n} Y_{n}^{(a+b)},} \\
& \left\{\left|Y_{m}^{(a)}, Y_{n}^{(b)}, Y_{p}^{(c)}\right|\right\}_{N}=Q^{(a, b, c)}{ }_{m n p}{ }^{\alpha} X_{\alpha}^{(a+b+c)} .
\end{aligned}
$$

Let $\rho$ be a $n$-dimensional representation of $\mathfrak{g}$ and let $M_{\alpha}^{(a)}=\rho\left(X_{\alpha}{ }^{(a)}\right), N_{m}^{(a)}=\rho\left(Y_{m}{ }^{(a)}\right)$ denote the corresponding transformed basic elements. Then the representation space $V$ on which the matrices $M$ and $N$ act satisfies the decomposition $V=\underset{a \in \Gamma}{\oplus} V_{0, a} \underset{a \in \Gamma}{\oplus} V_{1, a} \underset{a \in \Gamma}{\oplus} V_{2, a}$. Now, since the inclusions $M_{\alpha}^{(a)} V_{i, b} \subseteq V_{i, a+b}$,

\footnotetext{
${ }^{1}$ In particular, this way to construct representations does not preserve dimensions, as follows at once from the tensor products.

${ }^{2}$ The quon algebra originally introduced by Greenberg is real, such that the Fock space is a Hilbert space.

${ }^{3}$ The relations $a_{i} a_{j}-q a_{j} q_{i}=0$ only hold when the additional constraint $q^{2}=1$ is satisfied [5].
} 
$N_{m}^{(a)} V_{i, b} \subseteq V_{i+1, a+b}$ are satisfied, we can find a basis of $V$ such that $V=V_{0} \oplus V_{2} \oplus V_{1}$, i.e., with respect to the grading group $\mathbb{Z}_{3}$, the block $V_{i}=\operatorname{dim} \underset{a \in \Gamma}{\oplus} V_{i, a}$ is of degree $i$, for $i=0,1,2$. With respect to this basis, the matrices $M$ and $N$ can be rewritten as

$$
M_{\alpha}{ }^{(a)}=\left(\begin{array}{ccc}
M_{0 \alpha}{ }^{(a)} & 0 & 0 \\
0 & M_{2 \alpha}{ }^{(a)} & 0 \\
0 & 0 & M_{1 \alpha}{ }^{(a)}
\end{array}\right), \quad N_{m}{ }^{(a)}=\left(\begin{array}{ccc}
0 & M_{0-2 m^{(a)}} & 0 \\
0 & 0 & M_{2-1 \alpha^{(a)}} \\
M_{1-0 \alpha}{ }^{(a)} & 0 & 0
\end{array}\right) .
$$

Let $n_{i}=\operatorname{dim} V_{i}, i=1,2,3$, where obviously $n_{0}+n_{1}+n_{2}=n$. We denote by $v=\left(v_{i_{0}}, v_{i_{1}}, v_{i_{2}}\right)^{T}$ the components of the vector $v \in V\left(1 \leq i_{a} \leq n_{a}, a=1,2,3\right)$, and the matrix elements of $M$ and $N$ : $\left(M_{0 \alpha}{ }^{(a)}\right)_{i_{0}}^{{ }_{0}{ }^{0}},\left(M_{1 \alpha}{ }^{(a)}\right)_{i_{1}}^{j_{1}},\left(M_{2 \alpha}{ }^{(a)}\right)_{i_{2}}{ }^{j_{2}},\left(N_{0-2 m}{ }^{(a)}\right)_{i_{0}}{ }^{j_{2}},\left(N_{2-1 m}{ }^{(a)}\right)_{i_{2}}{ }^{j_{1}},\left(N_{1-0 m}{ }^{(a)}\right)_{i_{1}}{ }^{j_{0}}$. From now on, we adopt the convention that an index in the form $i_{a}, a=0,1,2$ is of degree $a$ with respect to the grading group $\mathbb{Z}_{3}$. Furthermore, using the same notations as in Section 2 with respect to the grading group $\Gamma$, $v_{i_{a}}$ is of degree $\operatorname{gr}\left(i_{a}\right)$. This in particular implies some relations for the matrix elements of $M$ and $N$. For instance, considering the matrix element $\left(N_{2-1 m}{ }^{(a)}\right)_{i_{2}}{ }^{j_{1}}$, we have $a=\operatorname{gr}\left(i_{2}\right)-\operatorname{gr}\left(j_{1}\right)$, etc. Consider now three series of quons $\mathcal{Q}_{n_{0}, 0}(\mathbb{C})=\left\langle a_{0 i}, a_{0}{ }^{i}, i=1, \cdots, n_{0}\right\rangle, \quad \mathcal{Q}_{n_{1}, 0}(\mathbb{C})=\left\langle a_{1 i}, a_{1}{ }^{i}, i=1, \cdots, n_{1}\right\rangle$ and $\mathcal{Q}_{n_{2}, 0}(\mathbb{C})=\left\langle a_{2 i}, a_{2}{ }^{i}, i=1, \cdots, n_{2}\right\rangle$ such that for any $m \neq n$, the relation $a_{m i} a_{n}{ }^{j}=0$ holds. It follows from the grading group $\mathbb{Z}_{3}$ that $a_{a}{ }^{i_{a}}$ (respectively $a_{a i_{a}}$ ) is of degree $a$ (resp. of degree $-a$ ), while, with respect to the group $\Gamma, a_{a}{ }^{i_{a}}$ (resp. $a_{a i_{a}}$ ) is of degree $\operatorname{gr}\left(i_{a}\right)$ (resp. $-\operatorname{gr}\left(i_{a}\right)$ ). We now define

$$
\begin{aligned}
& \mathcal{M}_{\alpha}{ }^{(a)}=\left(\begin{array}{lll}
a_{0}{ }^{i_{0}} & a_{2}{ }^{i_{2}} & a_{1}{ }^{i_{1}}
\end{array}\right)\left(\begin{array}{ccc}
\left(M_{0 \alpha}{ }^{(a)}\right)_{i_{0}}{ }^{j_{0}} & 0 & 0 \\
0 & \left(M_{2 \alpha}{ }^{(a)}\right)_{i_{2}} j_{2} & 0 \\
0 & 0 & \left(M_{1 \alpha}{ }^{(a)}\right)_{i_{1}} j_{1}
\end{array}\right)\left(\begin{array}{l}
a_{0 j_{0}} \\
a_{2 j_{2}} \\
a_{1 j_{1}}
\end{array}\right), \\
& \mathcal{N}_{m}{ }^{(a)}=\left(\begin{array}{lll}
a_{0}{ }^{i_{0}} & a_{2}{ }^{i_{2}} & a_{1}{ }^{i_{1}}
\end{array}\right)\left(\begin{array}{ccc}
0 & \left(M_{\left.0-2 m^{(a)}\right)_{i_{0}}{ }^{j_{2}}}\right. & 0 \\
0 & 0 & \left(M_{\left.2-1 m^{(a)}\right)_{i_{2}}{ }^{j_{1}}}\right.
\end{array}\right)\left(\begin{array}{l}
a_{0 j_{0}} \\
a_{2 j_{2}} \\
a_{1 j_{1}}
\end{array}\right) .
\end{aligned}
$$

By definition, the matrices $M_{\alpha}{ }^{(a)}$ and $N_{m}{ }^{(a)}$ satisfy the relations (5.2). Now, applying Lemma 5.1, the elements $\mathcal{M}_{\alpha}{ }^{(a)}, \mathcal{N}_{m}{ }^{(a)} \in \mathcal{Q}_{n_{0}, 0}(\mathbb{C}) \oplus \mathcal{Q}_{n_{1}, 0}(\mathbb{C}) \oplus \mathcal{Q}_{n_{2}, 0}(\mathbb{C})$ satisfy the same relations. Furthermore, since the quon algebra is an associative algebra, the Jacobi identities are automatically satisfied. Therefore the color algebra $\mathfrak{g}$ has been realized in the quon algebra $\mathcal{Q}_{n_{0}, 0}(\mathbb{C}) \oplus \mathcal{Q}_{n_{1}, 0}(\mathbb{C}) \oplus \mathcal{Q}_{n_{2}, 0}(\mathbb{C})$, finishing the proof. Ш It

should be observed that certain types of Lie algebras of order 3 do not admit finite dimensional matrix representations. However, these can realized by means of Clifford algebras of polynomials [14, 18]. Moreover, a similar argumentation allows to realize any given type of algebra admitting finite dimensional representations by an appropriate set of quons with $q=0$.

\section{Acknowledgments}

During the preparation of this work, the author (RCS) was financially supported by the research projects MTM2006-09152 (M.E.C.) and CCG07-UCM/ESP-2922 (U.C.M.-C.A.M.).

\section{References}

[1] Y. Bahturin, A. A. Mikhalev, V. M. Petrogradsky and M. V. Zaicev, Infinite-dimensional Lie superalgebras, de Gruyter Expositions in Mathematics, 7. Walter de Gruyter \& Co., Berlin, 1992.

[2] X.-W. Chen, S. D. Silvestrov, F. van Oystaeyen, Representations and Cocycle Twists of Color Lie Algebras. Algebras and Representation Theory, Algebr. Represent. Theory 9, 2006, no. 6, 633-650; H. Ljungqvist, S. D. Silvestrov, Involutions in three-dimensional coloured Lie algebras, Research Reports 6 , Dep. Math., Umea University, (1996), 36 pp; Ostrovskii, V. L.; Silvestrov, S. D. Representations of real 
forms of the graded analogue of a Lie algebra (Russian) Ukran. Mat. Zh. 44 (1992), no. 11, 1518-1524; translation in Ukrainian Math. J. 44 (1992), no. 11, 1395-1401 (1993) G. Sigurdsson, S. D. Silvestrov, Lie color and hom-Lie algebras of Witt type and their central extensions, in "Generalized Lie Theory in Mathematics, Physics and Beyond, Eds: S. Silvestrov, E. Paal, V. Abramov, A. Stolin, Spinger, 2009, 9pp. G. Sigurdsson, S. D. Silvestrov, Bosonic Realizations of the Colour Heisenberg Lie Algebra, J. of Nonlinear Mathematical Physics, 13 Supl. (2006), 110-128. G. Sigurdsson, S. D. Silvestrov, Graded quasi-Lie algebras of Witt type, Czechoslovak J. Phys.56, 11-12 (2006), 1287-1291. G. Sigurdsson, S. D. Silvestrov, Canonical involutions in three-dimensional generalised Lie algebras 50 (2000), no. 1, 181-186. Silvestrov, S. D. On the classification of 3-dimensional coloured Lie algebras, in "Quantum groups and quantum spaces (Warsaw, 1995), Banach Center Publ., 40, Polish Acad. Sci., Warsaw, 1997, 159-170. Silvestrov, S. D. Hilbert space representations of the graded analogue of the Lie algebra of the group of plane motions, Studia Math. 117 (1996), no. 2, 195-203.

[3] S. Coleman and J. Mandula, All Possible Symmetries of the S-Matrix, Phys. Rev. 159 (1967) B 12511256; O. W. Greenberg, Coupling of Internal and Space-Time Symmetries, Phys. Rev. 135 (1964) 1447-1450; L- O'Raifeartaigh, Lorentz Invariance and Internal Symmetry, Phys. Rev 139 (1965), B 1052-1062. [arXiv:hep-th/0312066].

[4] L. Corwin, Y. Ne'eman and S. Sternberg, Graded Lie Algebras in Mathematics and Physics (Bose-Fermi symmetry), Rev. Mod. Phys. 47 (1975), 573-603.

[5] D. I. Fivel, Interpolation between Fermi and Bose Statistics Using Generalized Commutators, Phys. Rev. Lett. 65 (1990) 3361-3364; O. W. Greenberg, Particles with small violations of Fermi or Bose statistics, Phys. Rev. D 43 (1991) 4111-4120; [arXiv:quant-ph/9903069].

[6] P. G. O. Freund, I. Kaplansky, Simple Supersymmetries, J. Math. Phys. 17 (1976), 228-231; R. Haag, J. T. Lopuszanski and M. Sohnius, All Possible Generators Of Supersymmetries Of The S Matrix, Nucl. Phys. B 88 (1975) 257-274.

[7] O. W. Greenberg, Example of Infinite Statistics, Phys. Rev. Lett. 64 (1990) 705-708.

[8] M. Goze and M. Rausch de Traubenberg, Hopf algebra for Ternary Algebras and Group arXiv:0809.4212 [math-ph].

[9] M. Goze, M. Rausch de Traubenberg and A. Tanasa, Poincaré and $\mathfrak{s l}(2)$ algebras of order 3, J. Math. Phys, 48 (2007) 093507. [arXiv:math-ph/0603008]; M. Rausch de Traubenberg and M. J. Slupinski, Fractional supersymmetry and $F(t h)$ roots of representations, J. Math. Phys. 41, (2000), 4556-4571 [arXiv:hep-th/9904126]; M. Rausch de Traubenberg and M. J. Slupinski, Finite-dimensional Lie algebras of order F, J. Math. Phys. 43, (2002), 5145-5160 [arXiv:hep-th/0205113].

[10] H. S. Green and P. D. Jarvis, Casimir Invariants, Characteristic Identities And Young Diagrams For Color Algebras And Superalgebras, J. Math. Phys. 24, (1983), 1681-1687.

[11] M. Günaydin and F. Gürsey, Quark statistics and octonions, Phys. Rev. D 9 (1974), 3387-3391.

[12] R. Kleeman, Aspects of modular quantization J. Math. Phys. 24 (1983), 166-172.

[13] J. Lukierski and V. Rittenberg, Color - De Sitter And Color - Conformal Superalgebras, Phys. Rev. D 18, (1978), 385-389; L. A. Wills-Toro, L. A. Sánchez, D. Bleecker, Trefoil symmetry. V. Class representations for the minimal clover extension, Internat. J. Theoret. Phys. 42 (2003), 73-83; L. A. Wills-Toro, L. A. Sánchez, X. Leleu, Trefoil symmetry. IV. Basic enhanced superspace for the minimal vector clover Internat. J. Theoret. Phys. 42 (2003), 57-72; L. A. Wills-Toro, Trefoil symmetry. III. The full clover extension, J. Math. Phys. 42 (2001), 3947-3964; L. A. Wills-Toro, L. A. Sánchez, J. M. Osorio, D. E. Jaramillo, Trefoil symmetry. II. Another clover extension, J. Math. Phys. 42 (2001), 39353946; L. A. Wills-Toro, Trefoil symmetries. I. Clover extensions beyond Coleman-Mandula theorem, J. Math. Phys. 42 (2001), 3915-3934. 
[14] N. Mohammedi, G. Moultaka and M. Rausch de Traubenberg, Field theoretic realizations for cubic supersymmetry, Int. J. Mod. Phys. A 19, (2004), 5585-5608 [arXiv:hep-th/0305172]; G. Moultaka, M. Rausch de Traubenberg and A. Tanasa, Cubic supersymmetry and abelian gauge invariance, Int. J. Mod. Phys. A 20, (2005), 5779-5806 [arXiv:hep-th/0411198]; G. Moultaka, M. Rausch de Traubenberg and A. Tanasa, Non-trivial extension of the Poincare algebra for antisymmetric gauge fields, Proceedings of the XIth International Conference Symmetry Methods in Physics, Prague 21-24 June 2004, arXiv:hepth/0407168.

[15] A. O. Morris, On a generalized Clifford algebra, Quart. J. Math., Oxford 18 (1967) 7-12; A. O. Morris, On a generalized Clifford algebra. II, Quart. J. Math., Oxford 19 (1968) 289-299. ; M. Rausch de Traubenberg, Clifford algebras, supersymmetry and $Z_{n}$ symmetries: Applications in field theory, arXiv:hep-th/9802141; M. Rausch de Traubenberg and N. Fleury, Linearization of polynomials J. Math. Phys. 33, (1992) 3356-3366.

[16] M. Rausch de Traubenberg, Four dimensional cubic supersymmetry, Pr. Inst. Mat. Nats. Akad. Nauk Ukr. Mat. Zastos., 50, Part 1, 2, 3, Natsional. Akad. Nauk Ukrani, Inst. Mat., Kiev, 2004, pp. 578-585.

[17] V. Rittenberg and D. Wyler, Sequences Of Z(2) Z(2) Graded Lie Algebras And Superalgebras, J. Math. Phys. 19, (1978), 2193-2200; V. Rittenberg and D. Wyler, Generalized Superalgebras, Nucl. Phys. B 139, (1978), 189-202.

[18] N. Roby, Algèbres de Clifford des formes polynomes, C. R. Acad. Sc. Paris, 268 (1969) A484-A486; Ph. Revoy, Nouvelles algb̀res de Clifford, C. R. Acad. Sc. Paris 284 (1977) A985-A988; N. Fleury, M. Rausch de Traubenberg, Linearization of polynomials, J. Math. Phys. 33 (1992) 3356-3366; N. Fleury and M. Rausch de Traubenberg, Finite-dimensional representations of Clifford algebras of polynomials Adv. Appl. Cliff. Alg. 4 (1994) 123-130; M. Rausch de Traubenberg, Habilitation Thesis, hep-th/9802141 [in French].

[19] M. Scheunert, Generalized Lie Algebras, J. Math. Phys. 20, (1979), 712-720. 